\title{
Consultorio de Atención Pediátrica Ambiental en un hospital de alta complejidad
}

\author{
Clinic of environmental pediatric care in a high complexity \\ hospital
}

\author{
Dra. Marisa Gaioli', Dr. Diego Amoedo y Bioq. Daniel González ${ }^{b}$
}

\begin{abstract}
RESUMEN
La Organización Mundial de la Salud considera la salud ambiental infantil como uno de los principales retos de la salud pública del siglo XXI y promueve el desarrollo de programas que permitan abordar, divulgar o mitigar el impacto en la salud de los contaminantes ambientales en todos los niveles de atención pediátrica.

El consultorio de Atención Pediátrica Ambiental (APA) que funciona en el Hospital de Pediatría "Prof. Dr. Juan P. Garrahan" se organizó con el objetivo de atender las demandas de aquellos pacientes con sospechada o comprobada exposición a riesgos ambientales. Se utilizó una historia clínica ambiental pediátrica, elaborada para tal fin.

Palabras clave: historia clínica ambiental, atención en consultorio, factores de riesgo ambiental.
\end{abstract}

\begin{abstract}
Children environmental health is considered by The World Health Organization as one of the main challenges of Public Health during the Century XXI. They promote the development of programs that allow approaching, disclosure or mitigation of the impact of polluting agents on health at every level of pediatric attention.

The Children Hospital "Prof. Dr. Juan P. Garrahan" has created an Environmental Health Office in order to address the demands of those patients with suspected or verified exposure to environmental risks. An Environmental Clinical History has been elaborated with this purpose. Key words: environmental clinical history, office practice, environmental health risk factors.
\end{abstract}

http:/ /dx.doi.org/10.5546/aap.2014.562

1. Consultorio Externo de Clínica.

2. Laboratorio de Toxicología y Absorción Atómica. Hospital de Pediatría SAMIC "Prof. Dr. Juan P. Garrahan".

Correspondencia: Dra. Marisa Gaioli: mgaioli@gmail.com

Conflicto de intereses: Ninguno que declarar.

Recibido: 31-12-2013

Aceptado: 25-6-2014
Las enfermedades pediátricas vinculadas con el ambiente constituyen un serio problema para la salud pública en el mundo. Los efectos adversos que pueden sufrir los niños, desde su concepción hasta la adolescencia, pueden manifestar su impacto en forma inmediata o a largo plazo, manteniéndose silentes hasta la adultez. ${ }^{1}$

Los factores ambientales constituyen más de la tercera parte de la carga global de enfermedades. En el año 2006, la OMS informó que más del $40 \%$ de dicha carga recaía en los menores de 5 años, quienes constituían el $10 \%$ de la población mundial. Además, informó que el $36 \%$ de las muertes entre 0 y 14 años podrían atribuirse al ambiente. ${ }^{3}$

Los niños se enfrentan cotidianamente a diferentes riesgos ambientales en los lugares donde viven, crecen, aprenden y juegan. La vulnerabilidad de esta población genera la necesidad de identificar los problemas ambientales relacionados con la calidad del agua, del aire, la seguridad de los alimentos, la exposición a metales pesados y a sustancias químicas peligrosas, las posibilidades de acceder a la educación y a la salud para que puedan alcanzar el máximo potencial de desarrollo y acceder legítimamente a sus sueños.

El ambiente físico es solo uno de los determinantes de la salud, y se denomina así a todos aquellos factores que influyen en la salud individual y que, interactuando en diferentes niveles de organización, determinan la salud de una población. Además, intervienen aquellos relacionados con el estilo de vida, la biología humana 
y el acceso a la atención de la salud. Por ello, es indispensable considerarlos en el momento de la anamnesis y evaluación clínica del niño y su familia relacionadas con la exposición ambiental.-6 (Figura 1).

\section{Experiencias en nuestro Consultorio de Atención Pediátrica Ambiental}

Atentos a la propuesta de la OMS y con el objetivo de dar respuesta a las numerosas consultas realizadas en el Hospital a propósito de la sospecha de exposición a factores ambientales adversos, en junio de 2011, se organizó en el Hospital de Pediatría "Prof. Dr. Juan P. Garrahan" un consultorio para la atención de pacientes que acudieran por sospecha de exposición a contaminantes ambientales o por intoxicaciones relacionadas con su entorno.

Los objetivos del Consultorio de Salud Ambiental Infantil son los siguientes:

1. Identificar los factores de riesgo ambientales a los que se encuentran expuestos los niños. Definir indicadores.

2. Asociar los factores de riesgo detectados con las manifestaciones clínicas del paciente, si las hubiera.

3. Contribuir a la vigilancia epidemiológica ambiental a través de la notificación obligatoria de aquellas enfermedades clasificadas como Enfermedades de Notificación Obligatoria (ENO), Sistema Nacional de Vigilancia de la Salud (SNSV) y a través del Sistema de Vigilancia de Laboratorio (SIVILA), según la Ley $15465 .^{7}$
4. Concientizar y capacitar a los actores en salud (a través de clases, ateneos, presentación de pacientes) sobre los riesgos ambientales reales y potenciales con la finalidad de iniciar estrategias de prevención, tanto individuales como comunitarias.

5. Informar a las familias sobre la adversidad del ambiente al que se encuentran expuestos a través de la concientización personalizada durante la consulta y también por medio de materiales gráficos elaborados por la Unidad Pediátrica Ambiental.

Ante la sospecha de exposición a factores de riesgo ambientales, los pacientes son derivados por profesionales de distintas áreas de nuestra Institución desde las siguientes áreas:

- Área de Bajo Riesgo.

- Área de Mediano Riesgo.

- Consultorios de clínica y de especialidades.

- Salas de Internación.

- Orientación Médica, donde consultan los pacientes que ingresan al Hospital por primera vez, ya sea en forma espontánea o derivados por sus médicos de cabecera.

La sospecha de exposición a factores ambientales adversos se puede expresar de variadas formas. Puede ser a través de la preocupación de los padres por la aparente contaminación del entorno, por ejemplo: cercanía a fábricas, parques industriales, asentamientos, presencia de vertederos, desarmaderos de autos, zonas inundables, basurales a cielo abierto, trabajo del padre (gasista o plomero), entre otras.

Figura 1. Determinantes sociales de la salud. Esquema de Marc Lalonde (1974) ${ }^{6}$

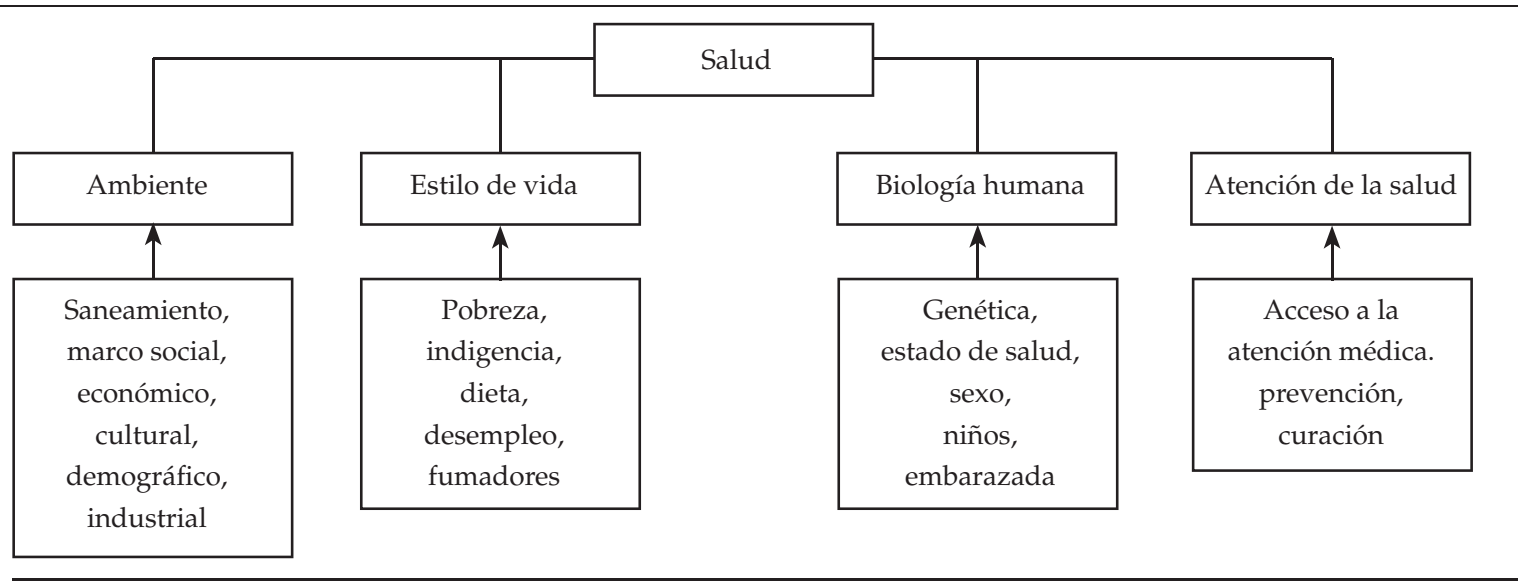


También pueden acudir ante la inquietud del médico de cabecera, quien sospecha la exposición considerando algunos determinantes sociales de la salud: inadecuado saneamiento ambiental, déficit de agua potable. Otra causa frecuente de consulta es por un cuadro clínico compatible con alguna intoxicación con un metal pesado (plomo, mercurio) o con arsénico. También el ámbito donde se encuentra emplazada la vivienda adquiere importancia: en el ámbito rural, es posible la exposición a plaguicidas; en una provincia del país, como en el caso de Santiago del Estero, zona endémica de hidroarsenicismo crónico, exposición al arsénico (As).

También los pacientes pueden ser derivados al consultorio cuando consultan por un dosaje de un metal pesado elevado, derivado desde otra institución o consultan en forma espontánea en busca de otra opinión.

El consultorio funciona semanalmente en el área de consultorios externos de clínica.

Los pacientes son atendidos por tres pediatras, con experiencia en Toxicología y especialistas en Salud y Ambiente, acompañados por un bioquímico especialista en Toxicología.

Considerando que nuestro Hospital no cuenta con Servicio de Toxicología propio, ante la necesidad de una consulta especializada, ésta se realiza telefónicamente con los Servicios de Toxicología del Hospital de Niños "Ricardo Gutiérrez" o del Hospital "Dr. Alejandro Posadas". Estas interconsultas se realizan frente a la necesidad de definir en conjunto el diagnóstico de un paciente con un cuadro clínico inespecífico en quien se sospecha una intoxicación o para compartir la decisión, por ejemplo, de iniciar terapia quelante en un paciente. Es importante destacar que no es necesaria la presencia de un médico toxicólogo para instalar un consultorio de Salud Ambiental Pediátrica, pero sí el estrecho contacto con ellos, ya sea por vía telefónica, por correo electrónico o fax.

El Laboratorio Central del Hospital de Pediatría Garrahan cuenta con un área especializada en toxicología de referencia nacional, donde se realizan los dosajes de diferentes tóxicos. Las pruebas de laboratorio que se han realizado, solicitadas a partir de este consultorio, han sido para confirmar o descartar diagnósticos. Entre ellas, plomo en sangre, arsénico en cabellos y uñas, arsénico, cromo y mercurio en orina de $24 \mathrm{~h}$. En algunos pacientes, fue necesaria la medición de colinesterasa (por sospecha de exposición a plaguicidas organofosforados), orto-cresol y ácido trans, trans-mucónico para evaluar la exposición a hidrocarburos y, en otros, fue necesario enviar las muestras a la Facultad de Farmacia y Bioquímica ante la sospecha de intoxicación con plaguicidas para su dosaje.

Con el objetivo de identificar los datos relacionados con los determinantes sociales de salud ambiental de los pacientes evaluados (Figura 1), se confeccionó una historia clínica basada en la Hoja Verde de la OMS y en la Hoja Pediátrica de Pesquisa del Riesgo Ambiental del Gobierno de la Ciudad Autónoma de Buenos Aires. ${ }^{1,8}$

Desde junio de 2011 hasta mayo de 2014, se atendieron 264 pacientes, de los cuales el $60 \%$ $(n=158)$ consultaron por sospecha de exposición a contaminantes ambientales y el $40 \%$, por intoxicaciones (el 92\% de estos últimos resultaron asintomáticos). En 105 casos, las intoxicaciones fueron atribuidas principalmente al plomo $(\mathrm{Pb})$ : 77\%; mercurio $(\mathrm{Hg})$ : 5\%; arsénico (As): $9 \%$; cobre $(\mathrm{Cu}): 2 \%$; y monóxido de carbono (CO): $7 \%$.

El mayor porcentaje de los pacientes proviene del Conurbano bonaerense: $78 \%(n=200) ; 10 \%$ $(\mathrm{n}=26)$, de la Ciudad Autónoma de Buenos Aires; $12 \%(n=31)$, del resto de la provincia de Buenos Aires; $2 \%(n=5)$, de otras provincias; y $1 \%(n=3)$, de países limítrofes.

De los niños evaluados, el $61 \%$ vive en ámbito urbano; 33\%, en el Conurbano bonaerense; y solo un $6 \%$, en ámbito rural. El $41 \%$ de los pacientes totales residen en el área de la Cuenca MatanzaRiachuelo.

El $15 \%$ de los pacientes $(n=39)$ presentan enfermedades de base: el 20\% $(n=7)$ corresponde a enfermedades complejas, considerando que las consultas se realizaron en un hospital de alta complejidad (aplasia medular, drepanocitosis, trasplante renal, trasplante de médula ósea, enfermedad de Gaucher, cardiopatías congénitas), y el $80 \%$ restante corresponde a otras enfermedades prevalentes de la infancia, como asma, bronquitis obstructiva, dermatitis atópica.

Sobre la base de datos obtenidos sobre el tipo de vivienda, el $47 \%$ de estas resultan adecuadas (construidas con material resistente y piso aislante). Un $18 \%$ de las familias vive en condiciones de hacinamiento y el 15\% tiene necesidades básicas insatisfechas (NBI).

Es importante destacar que el porcentaje de hogares con agua potable de suministro por red pública fue del $62 \%$, pero de ellas solo el $26 \%$ estaba conectado al sistema cloacal. Otras fuentes de provisión de agua consideradas 
fueron agua de pozo, recolección de agua de lluvia en tanques y aljibes.

Según el Registro de hogares con conexión a sistemas cloacales, el $88 \%$ de los evaluados no están conectados a redes cloacales. En referencia a factores socioculturales, el $96 \%$ de los familiares a cargo de los pacientes eran alfabetos. Se constató un caso de situación de trabajo infantil (el niño ayudaba a su padre en tareas rurales).

De los 264 pacientes evaluados, un $72 \%$ de sus familias presentaba preocupación por los problemas ambientales de la zona donde vivía. De las restantes, el 3\%, a pesar de presentar exposición real a contaminantes ambientales, no presentaba preocupación alguna por estos y había consultado por otras causas.

El estado nutricional de los pacientes era adecuado en un $98 \%$; solo el $2 \%$ respondía a grado I de desnutrición, y se trataba de pacientes portadores de cardiopatía congénita.

Con respecto al trabajo de los padres y/o convivientes, resultaron de riesgo ambiental o actividades con alta exposición a tóxicos ambientales el $40 \%$ de los evaluados, lo que constituyó la fuente de exposición de los niños y el resto de la familia. Estos se desempeñaban como plomeros, gasistas, recolectores urbanos, joyeros y en actividades de pesca como pasatiempo (con la fabricación de plomadas intradomicilio).

Considerando la utilización de posibles contaminantes domésticos de mayor peligrosidad, el 91,7\% utilizaba plaguicidas de origen domiciliario contra vectores, como mosquitos, cucarachas, moscas y roedores. En un caso, de ámbito de vida rural, la familia se encontraba expuesta a plaguicidas organofosforados sin conocer sus efectos nocivos. Se realizó capacitación de la familia y contacto con el centro de salud cercano y así se pudieron aplicar estrategias de prevención y minimización de riesgos.

El entorno a sus viviendas era bien conocido por el $99,8 \%$ de las familias.

Se registró la presencia de fábricas, basurales a cielo abierto, antenas, vertederos en un $72 \%$ de los casos. En algunos de ellos se realizaron evaluaciones junto con organizaciones no gubernamentales y, en otros, con la Secretaría de Salud del municipio al cual pertenecían.

La disposición final de la basura era adecuada en el $76 \%$ de los casos. El resto la arrojaba a basurales a cielo abierto $(12 \%)$ o la quemaba (12\%).

El 99\% de la población tenía acceso a un centro de salud cercano al domicilio.
El 84\% de la población evaluada se encontraba en riesgo de exposición a contaminantes ambientales, teniendo en cuenta diferentes determinantes sociales de la salud, como saneamiento básico deficiente, vivienda inadecuada, presencia de vectores peri o intradomicilio, o inadecuada disposición de basura.

\section{COMENTARIO}

El Consultorio de Atención Ambiental ha permitido identificar a los pacientes expuestos, a sus progenitores y a otros convivientes, e iniciar medidas de prevención o tratamiento oportuno en los pacientes intoxicados.

La mirada de la salud ambiental es holística con respecto a la salud y, por eso, el trabajo debe ser multidisciplinario.

Los Centros de Atención Primaria Ambiental (APA) gozan del beneficio de trabajar en un área programática determinada, con pleno conocimiento de los problemas ambientales locales, con un acceso directo a las familias y a las autoridades competentes para el planteo de gestiones y estrategias para minimizar o mitigar los riesgos de exposición. ${ }^{9}$

Sin embargo, teniendo en cuenta la importancia de identificar la numerosa población expuesta en la actualidad a condiciones ambientales adversas, es necesario implementar la evaluación de esos niños, cualquiera sea el nivel de atención, para arribar a un diagnóstico de certeza clínico y de laboratorio, y luego lograr la articulación con los centros de atención zonales para proteger a los niños, a su familia y el ambiente, y lograr el empoderamiento de las comunidades en el ámbito de la sostenibilidad local.

La historia clínica ambiental pesquisa minuciosamente el entorno donde el niño y su familia viven, permite conocer los determinantes sociales de la salud, la exposición a contaminantes ambientales reales o potenciales $\mathrm{y}$, a los trabajadores de la salud, realizar un diagnóstico de situación, formular una hipótesis y decidir acciones a través del seguimiento longitudinal del niño. Puede ser utilizada en pacientes sintomáticos o asintomáticos. Registra la percepción de riesgo ambiental por parte del familiar o adulto responsable que acompaña al niño a la consulta y evalúa las diferentes situaciones que pueden acontecer en el hogar, en la comunidad, en el área de trabajo, en la escuela y también conductas de riesgo y accidentes. ${ }^{1,8}$

En los pacientes evaluados en el consultorio, el diagnóstico de situación permitió enumerar los 
problemas, articularlos y proponer estrategias de gestión, tanto individuales como comunitarias, para minimizar los riesgos $y$, en caso de que fuera posible, mitigarlos.

La OMS informó, en el año 2006, que el 40\% de los niños menores de 15 años del mundo eran pobres y el 13\% indigentes. Así, en nuestra evaluación, la pobreza también resultó ser el mayor determinante de riesgo ambiental, expresada por viviendas inadecuadas, saneamiento básico deficitario o inexistente, falta de disponibilidad de trabajo o trabajos de riesgo de sus padres por exposición a contaminantes ambientales. ${ }^{1}$

La pobreza se relaciona directamente con las condiciones ambientales. El impacto del nivel socioeconómico-cultural afecta la salud de las familias. Los ingresos familiares tienen una influencia determinada en el ambiente en que el niño vive y las oportunidades que se le presentan. En las zonas más pobres, hay problemas de infraestructura de viviendas y mayor mortalidad. Estas familias se encuentran más expuestas a los contaminantes ambientales y padecen más su impacto sobre la salud. ${ }^{1,10}$

En un futuro próximo, sería beneficioso establecer un mapa de riesgo sanitario en relación con factores ambientales a través de un estudio multicéntrico con la finalidad de obtener información y registro acerca de la población infantil en riesgo real e inminente de exposición.

Todos los niños del mundo tienen derecho a crecer y desarrollarse en ambientes saludables donde puedan alcanzar sus sueños y desarrollar al máximo sus potencialidades.

Las autoridades gubernamentales, los profesionales de la salud, los educadores y las familias debemos proteger a los niños de las amenazas ambientales y garantizarles un ambiente donde ellos y sus generaciones puedan ser felices tanto en el presente como en el futuro.

\section{CONCLUSIONES}

Un Consultorio de Atención Pediátrica Ambiental es un eslabón más en la atención global del niño y su familia.

La historia clínica ambiental debe formar parte del interrogatorio en toda consulta pediátrica para poder así definir los determinantes de salud del paciente e iniciar gestiones de remediación o mitigación en su entorno, si los hubiera. ${ }^{1}$

Cada uno de los factores analizados a través de la historia clínica ambiental contribuye a formular un diagnóstico sobre la salud ambiental del niño, a elaborar una estrategia de acción con participación multisectorial, que minimiza o remedia los riesgos ambientales de exposición.

\section{Agradecimientos}

Al Dr. Marcelo Andrade, jefe de Clínica de Consultorios Externos de Clínica del Hospital de Pediatría "Prof. Dr. Juan P. Garrahan", quien nos brindó su apoyo y colaboración en la organización del Consultorio Pediátrico Ambiental, y a los profesionales del Laboratorio de Toxicología.

\section{REFERENCIAS}

1. Ministerio de Salud de la Argentina, Sociedad Argentina de Pediatría, Asociación Argentina de Médicos por el Medio Ambiente, Canadian Institute of Child Health. Perfil SANA 2007. Buenos Aires: Ministerio de Salud; 2007. Capítulo 4, Acciones para la protección de la salud y el ambiente. Disponible en: http:/ / www.msal.gov.ar / promin/publicaciones/pdf/4-capitulo-sana.pdf. [Acceso: 7 de julio de 2014].

2. Organización Mundial de la Salud. Ambientes saludables y prevención de enfermedades. Hacia una estimación de la carga de morbilidad atribuible al ambiente. Ginebra, 2006. Disponible en: http://www.who.int/quantifying_ ehimpacts/publications/prevdisexecsumsp.pdf. [Acceso: 27 de junio de 2014].

3. Asociación Argentina de Médicos por el Medio Ambiente; International Society of Doctors for the Environment, InternationalNetwork on Children's HealthEnvironmentand Safety. El niño y su ambiente. Guía de consejos para proteger a los niños de los peligros ambientales. Santa Fe, Argentina: AAMMA, 2008. Disponible en: http://www.aamma.org/ wp-content/uploads/2009/05/el-nino-y-su-ambiente-114-2008-borrador-2.pdf. [Acceso: 7 de julio de 2014].

4. Quiroga D, Frack S. El ambiente, el pediatra y los niños. En Quiroga D, Fernández R, Paris E, comp. Salud Ambiental Infantil: manual para enseñanza de grado en escuelas de medicina. Buenos Aires: Ministerio de Salud de la Nación, Organización Panamericana de la Salud; 2010. Págs.9-13.

5. Ordoñez GA. Salud ambiental: conceptos y actividades. Rev Panam Salud Publica 2000;7(3):137-47.

6. Lalonde M. Social values and public health. Can J Public Health 1974;65(4):260-8.

7. Argentina. Ministerio de Salud de la Nación. Dirección de Epidemiología. Área de Vigilancia. Guía para el fortalecimiento de la Vigilancia de la Salud en el nivel local. Buenos Aires: Ministerio de Salud, 2013. Disponible en:http: / /www.msal.gov.ar/images/stories / epidemiologia/pdf/guia-c2_vigilancia.pdf. [Acceso: 7 de julio de 2014].

8. Ciudad de Buenos Aires. Ministerio de Salud. Hoja pediátrica de pesquisa del riesgo ambiental. Buenos Aires, 2014. Disponible en: http://www.buenosaires.gob.ar/ areas/salud/saludambiental/riesgos_ambientales/hoja_ pediatrica.php. [Acceso: 27 de junio de 2014].

9. Centro Panamericano de Ingeniería Sanitaria y Ciencias del Ambiente. División Salud y Ambiente OPS/OMS. Guía básica para la promoción de la atención primaria ambiental (APA). Washington: OPS, 2001. Disponible en: http:// www.bvsde.ops-oms.org/bvsaap/e/fulltext/guia/guia. pdf. [Acceso: 7 de julio de 2014].

10. Goldman LR. The clinical presentation of environmental health problems and the role of the pediatric provider. What do I do when I see children who might have an environmentally related illness? Pediatr Clin North Am 2001;48(5):1085-98,vii. 